Meta

Journal des traducteurs

Translators' Journal

\title{
Aspects culturels de la traduction : quelques notions clés
}

\section{Jean-Louis Cordonnier}

Volume 47, numéro 1, mars 2002

URI : https://id.erudit.org/iderudit/007990ar

DOI : https://doi.org/10.7202/007990ar

Aller au sommaire du numéro

\section{Éditeur(s)}

Les Presses de l'Université de Montréal

\section{ISSN}

0026-0452 (imprimé)

1492-1421 (numérique)

Découvrir la revue

Citer cet article

Cordonnier, J.-L. (2002). Aspects culturels de la traduction : quelques notions clés. Meta, 47(1), 38-50. https://doi.org/10.7202/007990ar

\section{Résumé de l'article}

La problématique de la culture constitue désormais un champ de recherche primordial pour travailler à une théorie de la traduction. On se situe ici au niveau du sol archéologique, c'est-à-dire au niveau des modes d'être de la culture, et de leurs interactions avec les modes de traduire. La traduction n'étant jamais une opération neutre, il convient de mettre en évidence les interventions du traducteur réalisées dans le cadre de son appartenance à telle ou telle culture. Mais il ne faut pas non plus réifier la culture, et il faut mettre en relief également les interventions d'ordre purement individuel. Cette relation à la culture est d'une grande importance puisque le traducteur, étant au coeur des relations d'altérité, constitue de par son activité traduisante, l'identité de sa propre culture. Il s'agit de passer aujourd'hui d'un ethnocentrisme négatif, procédant à l'effacement de l'Autre, à un ethnocentrisme positif réalisant par la " montre » de l'Autre, la tâche de constitution de l'identité propre. Ce dévoilement pour l'identité passe par la critique de la dichotomie par trop simpliste " cibliste/sourcier ", qui est prisonnière de la langue. Le traducteur se donnera en revanche comme tâche, la « montre » du discours de l'Autre. Cette problématique interculturelle est examinée à travers cinq champs clés dans lesquels se déploie l'activité traduisante : altérité, histoire, critique, éthique et tâches de la traduction.
Ce document est protégé par la loi sur le droit d'auteur. L’utilisation des services d'Érudit (y compris la reproduction) est assujettie à sa politique d'utilisation que vous pouvez consulter en ligne.

https://apropos.erudit.org/fr/usagers/politique-dutilisation/ 


\title{
Aspects culturels de la traduction: quelques notions clés
}

\author{
JEAN-LOUIS CORDONNIER \\ Université de Franche-Comté, Besançon, France
}

\begin{abstract}
RÉSUMÉ
La problématique de la culture constitue désormais un champ de recherche primordial pour travailler à une théorie de la traduction. On se situe ici au niveau du sol archéologique, c'est-à-dire au niveau des modes d'être de la culture, et de leurs interactions avec les modes de traduire. La traduction n'étant jamais une opération neutre, il convient de mettre en évidence les interventions du traducteur réalisées dans le cadre de son appartenance à telle ou telle culture. Mais il ne faut pas non plus réifier la culture, et il faut mettre en relief également les interventions d'ordre purement individuel. Cette relation à la culture est d'une grande importance puisque le traducteur, étant au cœur des relations d'altérité, constitue de par son activité traduisante, l'identité de sa propre culture. II s'agit de passer aujourd'hui d'un ethnocentrisme négatif, procédant à l'effacement de l'Autre, à un ethnocentrisme positif réalisant par la «montre» de l'Autre, la tâche de constitution de l'identité propre. Ce dévoilement pour l'identité passe par la critique de la dichotomie par trop simpliste «cibliste/sourcier», qui est prisonnière de la langue. Le traducteur se donnera en revanche comme tâche, la «montre» du discours de l'Autre. Cette problématique interculturelle est examinée à travers cinq champs clés dans lesquels se déploie l'activité traduisante: altérité, histoire, critique, éthique et tâches de la traduction.
\end{abstract}

\section{ABSTRACT}

The issue of culture is now a primary field of research in developing a theory of translation. Here we find ourselves on "archeological terrain" in terms of the culture's way of being and the concomitant interaction with ways of translating. Translation is never a neutral operation, and one is forced to demonstrate that the act of translating is influenced by the translator's cultural background. Culture, however, must not be reified; purely individual interventions must also be given their due. This relation to culture is highly important for the translator who is at the heart of relations of otherness, and through the act of translation it forms the identity of national culture. This entails moving from negative ethnocentrism, which erases the Other, to positive ethnocentrism, showing the Other, and thus constitutes the identity of the translator's culture. This unveiling of identity undergoes criticism by the too simplistic "cibliste/sourcier" dichotomy, which is a prisoner of language. The translator, however, shows the discourse of the Other. These intercultural issues are examined under five translating-as-activity headings: otherness, history, criticism, ethics and translating tasks.

\section{MOTS-CLÉS/KEYWORDS}

altérité, histoire, éthique, l'Autre

Nous commencerons par une remarque liminaire qui paraîtra une évidence pour le spécialiste, mais qu'il convient à notre avis de réaffirmer toujours et encore, tant on entend répéter par la tradition et l'empirisme, de colloque en colloque, voire d'article en article, des arguments annexionnistes justifiant une certaine intraduisibilité, tels que: «ça ne sonne pas français», ou «ça sent la traduction», ou encore «le lecteur ne 
comprendra pas », arguments entièrement situés dans la langue, qui par conséquent ne voient pas le discours (au sens de Benveniste), et inhibiteurs quant aux potentialités du travail de ré-écriture dans l'opération traduisante. Cette remarque, c'est que la traduction n'est pas seulement une opération linguistique, mais qu'elle est tout entière prise dans un ensemble d'interrelations sociales et culturelles, d'abord au sein de sa propre culture, et ensuite entre les cultures étrangères en présence. Les paramètres culturels sont à même de jouer par conséquent un grand rôle dans la traduction en général, y compris dans ce qu'on appelle traditionnellement la traduction scientifique et technique, même si ce type de traduction n'est pas le lieu où les enjeux culturels se manifestent avec le plus d'acuité.

Saluons donc l'initiative de nos collègues de l'Université technique de Yildiz, d'avoir consacré un colloque entier sur les Aspects culturels de la traduction. De telles rencontres, entièrement consacrées à ce thème, somme toute, n'étaient pas si fréquentes jusqu'à il y a peu ${ }^{1}$. Nous rappellerons qu'il y a presque déjà une quarantaine d'années maintenant que G. Mounin, dans ses Problèmes théoriques de la traduction, mettait en avant dans son chapitre XIII ce qu'il appelait un fait théorique, énoncé ainsi: "pour traduire une langue étrangère, il faut remplir deux conditions, dont chacune est nécessaire, et dont aucune en soi n'est suffisante : étudier la langue étrangère; étudier (systématiquement) l'ethnographie de la communauté dont cette langue traduite est l'expression. Nulle traduction n'est totalement adéquate si cette double condition n'est pas satisfaite » (Mounin 1963: 236). Naturellement, il est souhaitable de faire appel également à d'autres sciences humaines pour travailler à une traductologie aux multiples facettes, et nous pensons en particulier à la littérature, à l'histoire, aux sciences du langage, à l'anthropologie, à la sociologie, à la psychanalyse, à la philosophie.

Il faut dire que depuis 1963, date de la publication des Problèmes théoriques, l'appel de G. Mounin a tardé à se faire entendre, et si nous disposons de nombreux articles qui traitent de problèmes ponctuels en relation aux aspects culturels de la traduction, nous avons par contre peu d'ouvrages qui aient tenté d'embrasser la problématique culturelle dans son ensemble, et de la mettre en relation avec les autres problématiques du traduire. Cependant, en France, H. Meschonnic (1973) en forgeant dans les années soixante-dix le concept de «langue-culture» dans le cadre de sa poétique, a voulu indiquer qu'une langue et sa culture forment un tout indissociable. Les analyses de H. Meschonnic sont assez connues pour que nous n'y insistions pas, mais nous voulions juste signaler ici sa volonté de penser la traduction dans un vaste cadre culturel comprenant l'histoire, la littérature, le langage et le politique. Puis, en 1984, un ouvrage d'A. Berman a frappé l'attention des traducteurs et des traductologues, en montrant le rôle qu'un mouvement culturel tout entier, en l'occurrence celui imaginé par les Romantiques allemands, peut assigner à la traduction. De notre côté, nous avons essayé de placer la traduction au sein de la problématique culturelle. En montrant tout l'intérêt qu'il y a à développer une archéologie de la traduction et à situer la pratique du traduire dans le cadre d'une éthique, nous avons donné une vue synthétique, mais non exhaustive, des problèmes que les traducteurs rencontrent dans la traduction des œuvres, notre objectif consistant aussi à indiquer des directions de travail et de recherche (Cordonnier 1995).

Dans les limites du présent exposé, nous souhaitons apporter des précisions sur cinq notions clés, qui sont aussi cinq champs clés, autour desquels il nous paraît 
souhaitable que se développent dans les temps à venir à la fois le travail de mise en valeur sociale de l'activité traductive, et celui de la réflexion traductologique: altérité, histoire, critique, éthique et tâches de la traduction ${ }^{2}$. Les considérations qui vont suivre concernent les œuvres, c'est-à-dire ces textes qui créent, qui représentent l'essence d'une culture, qui en constituent les racines, et qui par le travail d'écriture qui les traverse, créent du discours (toujours au sens de Benveniste), redéploient la culture vers d'autres horizons désormais élargis et la grandissent. Il s'agit d'une précaution méthodologique pour ne pas être enfermé dans ce qu’on appelle traditionnellement «la littérature». C'est pourquoi nous pouvons inclure dans notre champ de recherche des œuvres appartenant aussi au domaine scientifique, dans la mesure où nous sommes confrontés à des textes où se manifeste une poétique qui est l'œuvre d'un sujet-écrivain (au sens de H. Meschonnic), c'est-à-dire une poétique qui n'appartient qu'à lui.

L'intitulé de notre colloque laisse un large champ des possibles quant à la compréhension du concept de culture. Par «aspects culturels», on peut entendre les connotations et les traits culturels, et le problème de leur importation dans la langue de traduction. Ce ne sera pas notre préoccupation aujourd'hui. On peut comprendre aussi, et c'est dans ce sens que nous irons pour notre part dans cet exposé, ce qui fait que la culture intervient dans les modes de traduire ${ }^{3}$, la traduction étant une opération éminemment culturelle, en ce sens qu'on ne traduit pas dans toutes les cultures de la même façon, et qu'il y a une interaction entre les modes de traduire et les modes d'être des cultures. En outre, la traduction n'est pas une activité isolée, mais elle se déploie en articulation avec d'autres genres essentiels qui président au destin des œuvres, comme la critique, le commentaire, l'analyse. Elle fait donc partie en ce sens de toute une tradition culturelle liée à la structuration de l'essence d'une culture.

Le concept de culture est complexe $e^{4}$. En ce qui nous concerne, nous nous référons ici au sens aujourd'hui très étendu de modes de vie et de pensée communs à une communauté donnée et qui conduisent les individus appartenant à cette communauté à agir dans certaines situations sociales d'une façon commune. C'est-à-dire que nous nous référons à ce que Michel Foucault (1966) a appelé les "modes d'être» d'une culture. Il convient de s'intéresser à ces modes d'être, dans la mesure où ils peuvent induire chez les traducteurs, des modes de traduire relativement communs à certains moments de la constitution des cultures, modes de traduire liés aux contraintes sociales qui pèsent sur eux. À ce propos nous pensons à la formation linguistique, culturelle, politique, des États-Nations en Europe. Nous situons notre réflexion dans le cadre de la culture française, et d'une façon plus large dans celui de la culture occidentale, dans la mesure où en Europe les États-Nations ont tous constitué leur prose et leur littérature sur la base de traductions.

D'un autre côté, et c'est le deuxième aspect de la notion de culture auquel nous nous référons aujourd'hui, il faut être attentif à ne pas réifier la culture, car elle n'est qu'une abstraction, une construction intellectuelle (voir Cuche 1996: 57). Il faut donc tenir compte également des pratiques individuelles, et notamment, thème que nous retenons ici, de la position du traducteur dans sa relation d'altérité face à l'Étranger, et de la conception qu'il a du rôle que doit jouer sa propre culture dans les rapports d'altérité. C'est donc cette dialectique entre la culture et l'individu, c'est-à-dire pour nous, le traducteur, qui sera le cadre de notre exposé. 


\section{Altérité}

La traduction se déployant au sein des rapports d'altérité, le traducteur se trouve devant la tâche d'avoir à importer des valeurs, des faits culturels, mais ce n'est pas là son seul rôle: "le traducteur n'est pas uniquement prospecteur des différences, explorateur de territoires culturels inconnus. Il est aussi celui qui, dans sa reconnaissance de l'autre, change les perspectives de sa communauté, dérange les «mots de sa tribu», pour reprendre l'expression fameuse de Mallarmé (1877). [...] Par delà les décideurs (commanditaires, éditeurs, etc.), par delà la matérialité des textes, [...] il brouille les cartes, en l'occurrence ces cultures, ces valeurs, celles de l'autre comme les siennes propres qu'on voudrait bordées, délimitées, alors qu'elles sont fluides, mouvantes» (Delisle et Woodsworth 1995: 193). Il y a deux idées fondamentales dans ce passage. La première est que le traducteur joue un rôle essentiel dans la constitution de sa propre culture. Autrement dit, il déstructure, façonne, restructure l'identité de sa propre culture, et à travers les textes traduits, celle de la culture étrangère. La deuxième idée est que toute culture, quelle qu'elle soit, n'est pas un tout absolument stable, figé, mais elle est un ensemble divers et complexe caractérisé par de constantes évolutions.

Nous reviendrons sur la question de l'identité quand nous aborderons plus loin la problématique de l'éthique. Mais ce que nous retiendrons pour l'instant, c'est à la fois le rôle de la culture ainsi que celui du traducteur dans les rapports d'altérité, rôles qu'il convient d'examiner plus précisément. Il n'est donc pas inutile de regarder quelle a été l'évolution de la notion de culture. Dans le cadre limité qui est ici le nôtre, nous nous contenterons de quelques remarques.

Nous avons tenté de montrer dans Traduction et culture ${ }^{5}$ en quoi en France l'épistémè de l'âge moderne, puis de l'âge classique, ne pouvaient concevoir la différence de l'Étranger dans toute sa radicalité, ce qui a conduit à la pratique de la traduction ethnocentrique, étant entendu que ce type de traduction a servi, et c'est là son rôle fécondateur et positif pour nous, à constituer la prose, et parallèlement la culture de notre pays.

En France, le $\mathrm{XVII}^{\mathrm{e}}$, puis le $\mathrm{XVIII}^{\mathrm{e}}$ siècle ont développé une conception universaliste de la culture, associée à la notion de civilisation. Les Lumières ont voulu propager ce qu'ils considéraient comme «leurs» bienfaits; dans leur esprit il s'agissait d'en faire bénéficier les autres peuples. Et cela était possible parce que s'était développée l'idée d'une unité du genre humain. En outre, le classicisme français est en Europe celui qui dure le plus longtemps, plus de deux siècles et demi. Au XIX ${ }^{\mathrm{e}}$ siècle, il y a à la fois un élargissement et une continuité de la notion de culture: "Entre le XVIII ${ }^{\mathrm{e}}$ et le $\mathrm{XIX}^{\mathrm{e}}$ siècle français, il y a une continuité de la pensée universaliste. La culture au sens collectif, c'est avant tout la «culture de l'humanité». Malgré l'influence allemande, l'idée d'unité l'emporte sur la conscience de la diversité [...]», et plus loin «En bonne logique, l'idée universaliste française de la culture va de pair avec la conception élective de la nation, issue de la Révolution: appartiennent à la nation française, expliquera Renan, tous ceux qui se reconnaissent en elle, quelles que soient leurs origines» (Cuche 1996: 13). C'est donc d'une certaine façon l'universalisme des Lumières qui, en ne pensant la diversité culturelle qu'en référence à la nation et à la civilisation, a conduit à une spécificité française: «Il est clair que le contexte idéologique propre à la France du $\mathrm{XIX}^{\mathrm{e}}$ siècle a bloqué l'émergence du concept descriptif 
de culture. Sociologues et ethnologues étaient eux-mêmes trop imprégnés de l'universalisme abstrait des Lumières pour penser la pluralité culturelle dans les sociétés humaines autrement qu'en référence à «la» civilisation. Le contexte historique, il est vrai, ne portait pas à l'interrogation sur cette question. L'épopée coloniale se faisait au nom de la mission «civilisatrice» de la France» (ibid.: 23). Denys Cuche ajoute qu'il faudra attendre les années trente pour que le concept de culture commence à être vraiment utilisé par les ethnologues. Il restera longtemps en concurrence avec la notion de civilisation, et il lui faudra encore trente années pour émerger définitivement et prendre une place indiscutable en ethnologie et en anthropologie (ibid.).

De ce qui précède il faut en déduire la nécessité de souligner le continuum historique, l'existence jusqu'à il y a peu d'un relatif impensé de la notion de différence culturelle, et en même temps de son rôle pour féconder, faire évoluer l'identité. Il s'agit là d'un simple constat. Dans le domaine de la traduction, on est loin d'avoir tiré toutes les conséquences, tant sur le plan de l'importation des traits culturels que sur celui de l'évolution de la ré-écriture dans les textes traduits, de l'examen scientifique et approfondi de la différence culturelle. Si la traduction est communication interculturelle, elle communique des contenus d'information, elle communique des spécificités culturelles, c'est-à-dire ce qui caractérise l'Autre et pas le Même, mais elle communique aussi par ce qu'elle est, nous voulons dire par là que la façon dont elle se pratique, ce que nous avons appelé les modes de traduire, apporte des informations sur l'être du traducteur et de sa culture dans son rapport à l'Autre.

C'est pourquoi nous avons appelé à une archéologie de la traduction, pour mettre à nu les modes de traduire et partant les modes d'être des traducteurs. Il convient d'insister sur l'ampleur de la tâche et sur les «trous» de connaissance, notamment en ce qui concerne le $\mathrm{XIX}^{\mathrm{e}}$ et le $\mathrm{Xx}^{\mathrm{e}}$ siècle, pour lesquels nous manquons d'ouvrages de synthèse. Ce travail contribuera probablement à montrer qu'il n'y a pas d'absolu en matière de traduction, et que sa pratique, la réflexion qui se développe autour d'elle changent en même temps que la culture change, traduction et culture étant toutes deux prises dans l'évolution de l'Histoire. Ainsi la pratique de la traduction au $\mathrm{XVI}^{\mathrm{e}}$ n'est pas la même que celle de l'âge classique, qui à son tour n'est pas la même que celle du XIX ${ }^{e}$ siècle, etc. D'un autre côté, une archéologie montre comment une culture dans son ensemble peut orienter, canaliser les pratiques. Ainsi, les traducteurs classiques, enfermés en quelque sorte dans la théorie de la représentation. À ce propos, Daniel Mercier montre bien comment, malgré des différences qui peuvent a priori sembler importantes, les traducteurs de l'âge classique sont tous obligés de situer leur pratique dans le cadre de la représentation classique, y compris Diderot qui a pourtant su mener la critique de la disposition classique, et donc de pratiquer un type de traduction qui naturalise, désécrit, annexe, nationalise (Mercier 1995). Il y a donc dans ce cas un parallèle entre la culture qui fonde un rapport universaliste à l'Étranger, sur ses propres critères culturels, et la pratique annexionniste de la traduction.

Nous disions plus haut que la traduction est communication interculturelle. Elle joue un rôle majeur dans l'altérité au niveau de la circulation des textes dans le monde. C'est là un phénomène bien connu. Ce qui l'est moins, c'est de savoir comment ces textes circulent, ce qu'on en fait, et ce qu'ils deviennent d'une culture à l'autre. Nous reprenons ici le concept de translation, développé par Antoine Berman (1995: 17). Il s'agit d'un espace d'échange entre les cultures en présence, où à côté du 
texte original, circulent dans un rapport dialectique, "les nombreuses formes de transformations textuelles (ou même non textuelles) qui ne sont pas traductives» (ibid.) : critiques, analyses, commentaires, films, adaptations, etc., dans l'une ou l'autre des cultures. Les rapports d'altérité s'exercent en ce lieu dans lequel se confrontent les conceptions du traducteur et aussi celles de chacune des cultures en présence au sujet de la littérature. Toutes ces interactions ne peuvent rester sans influence sur l'opération traduisante elle-même. Tout cet ensemble de mouvements textuels, lorsqu'ils se produisent, constituent ce qu'Antoine Berman appelle la «translation d'une œuvre»(ibid.). Cette notion nous apparaît très féconde dans la mesure où elle élargit la notion de traduction, qui souffrait d'étroitesse, à tout son espace littéraire naturel. Antoine Berman voit juste quand il affirme: "une traduction n'agit vraiment dans cette langue-culture que si elle est étayée et entourée par des travaux critiques et des translations non traductives» (ibid.: 18).

Mais il ne faut pas oublier d'ajouter dans l'étayage traductif, l'ensemble des textes qui entourent l'œuvre elle-même dans la langue-culture de l'Autre, et dont le traducteur est à même de prendre connaissance. En quoi l'altérité joue-t-elle dans cette confrontation des textes? Il y a là un sujet d'étude qui ne peut qu'être fécond, et apporter des informations importantes sur l'influence que peut exercer la culture étrangère sur le traducteur et sur son mode de traduire, éventuellement sur sa ré-écriture.

\section{Histoire}

Aujourd'hui il faut recourir à l'ensemble des outils conceptuels mis à notre disposition par l'ethnologie, l'anthropologie, la sociologie, la littérature, et qui nous permettront d'analyser sérieusement les paramètres de la culture dans la traduction. Cela est vrai en ce qui concerne les traductions de maintenant. Mais cela est plus difficile dès que l'on se tourne vers le passé, en raison des «trous » de connaissance auxquels nous avons fait référence, quoique certaines périodes, comme celle des belles infidèles aient assez largement inspiré les analystes ${ }^{6}$.

Quoiqu'il en soit, il est de première importance que se constituent une histoire, des histoires, de la traduction. Nous avons en effet un grave manque dans ce domaine. L'histoire de la traduction permettra de sortir les traducteurs de l'ombre, de mettre en exergue leur rôle au cœur des relations interculturelles, leur rôle de passeurs d'informations en tous genres, leur rôle de constitution des proses et des cultures nationales, leur rôle parfois de médiateurs, tout cela sans être exhaustif. Bref, le travail de recherche historique aidera à redonner au traducteur toute son importance au sein des cultures, et à renverser cet état d'effacement où il se trouve en France depuis la fin du $\mathrm{XvI}^{\mathrm{e}}$ siècle. Il s'agit donc de renverser cette situation de «secondarité» et d' "ancillarité» (Berman 1984) dans laquelle le surgissement de la figure de l'auteur a plongé la traduction, en faisant une activité seconde, dans laquelle le traducteur devait s'effacer pour ne laisser voir au lecteur qu'un double, tâcheron et sans âme.

En outre, la constitution d'une histoire, ou d'histoires de la traduction, représente l'une des tâches nécessaires pour que l'on puisse élaborer à terme ce qui est peut-être une inaccessible théorie de la traduction et de la littérature. Mais au moins aller vers. En 1995, le président de la Fédération internationale des traducteurs, JeanFrançois Joly, parlant justement de l'histoire de la traduction voyait que «cette jeune 
discipline ne saurait prétendre avoir un avenir si elle ne peut pas se nourrir des acquis du passé, se ressourcer à des modèles anciens. Faire l'histoire de la traduction, c'est mettre au jour le réseau complexe des échanges culturels intervenus entre les êtres humains, les cultures, les civilisations au cours des âges» (Delisle et Woodsworth 1995 : 15). Et il ajoutait, citant Lieven D’hulst, «l'histoire est pratiquement le seul moyen de retrouver l'unité d'une discipline, en montrant les parallèles et les recoupements entre les traditions de pensée et d'activité divergentes, en rapprochant le passé et le présent» (ibid.).

S'il est tout à fait vrai que l'histoire de la traduction peut introduire une unité dans la réflexion traductologique, nous pensons d'une part qu'elle n'est pas le seul moyen parce que la traduction se trouve à un carrefour des sciences humaines, et d'autre part ce n'est pas parce qu'il y a actuellement une heureuse focalisation des préoccupations traductologiques sur l'histoire, qu'il faille négliger pour autant les axes de recherches liés à l'anthropologie, à la linguistique ou à la philosophie, pour ne prendre que ces trois exemples. On ne pourra trouver une unité de la traduction qu'en l'étudiant sous tous ses aspects. C'est précisément cela qui fait qu'une traductologie puisant dans les sciences humaines une incontestable autorité, a du mal à se constituer. C'est d'ailleurs, à notre avis, plus le manque de moyens humains que scientifiques qui en constitue la difficulté.

D’un autre côté, il y a un risque à considérer, comme le pense Lieven D’hulst, que l'histoire de la traduction serait "pratiquement le seul moyen de retrouver l'unité» de la réflexion en matière de traduction. D'abord peut-on parler d'unité en histoire? Il faut commencer par poser ce problème méthodologique. En effet, il n'y a pas une seule méthodologie de l'histoire. Il n'y a donc pas de raison de ne pas retrouver en traduction les débats, voire les polémiques, que l'on rencontre chez les historiens. Ensuite, en traduction il y a un deuxième risque à vouloir faire de l'histoire pour de l'histoire, en l'absence d'une méthodologie scientifique rigoureuse, et en perdant de vue que la traduction est avant tout une activité interculturelle.

Nous voudrions illustrer ce dernier point par un exemple parlant. Dans Les traducteurs dans l'histoire, se trouve un article intitulé James Evans chez les Indiens cris $d u$ Canada (ibid. : 32-35) $)^{7}$. Cet article raconte comment ce missionnaire-traducteur a inventé un alphabet pour transcrire la langue de ce peuple. Inutile de dire que ce travail s'est déroulé dans le cadre beaucoup plus vaste de l'évangélisation de ces Indiens. Selon nous, cet article pose un problème important et souffre d'un manque grave. Il part en effet de l'a priori que le travail du traducteur chez ces Indiens est «bon» par définition, comme si l'évangélisation allait de soi. Les Indiens cris sont étrangement absents de ce récit et les conséquences sur leurs propres mythes, et de l'évangélisation, et de l'alphabet, ne sont absolument pas évoquées. Dans un cas comme celui-là l'historien doit se doubler d'un ethnologue. Il y a là une question primordiale, qui est débattue en ethnologie et en anthropologie: où se trouve la place de l'historien dans la relation interculturelle? Chez le Même? Chez l'Autre? Au milieu? Nous ne pouvons pas développer ici une réponse à cette question, mais dans cet exemple l'Indien cri aurait pu pour le moins être convoqué. En effet, il ne s'agit pas d'encenser le traducteur pour le seul fait qu'il soit traducteur, même si le travail accompli est remarquable, ou alors on se trouve dans une relation à sens unique, que pour notre part nous croyions dépassée. Bien sûr, il s'agit aussi d'interroger les modes de traduire du traducteur. 
Concernant Les traducteurs dans l'histoire, nous ne voudrions pas rester sur cette note critique, car il faut saluer cette entreprise et les énergies rassemblées par la FIT notamment, pour que vive ou plutôt commence à vivre, comme le précisent bien les directeurs de l'ouvrage dans leur Avant-propos, une histoire de la traduction. Du reste, le titre lui-même indique bien qu'il ne s'agit pas d'une histoire à proprement parler, mais de pistes qui ont été choisies dans le monde entier pour montrer le rôle des traducteurs, et peut-être pour susciter des envies d'approfondissement.

\section{Critique}

Pour constituer la traductologie ou la théorie de la traduction de demain, il y a tout intérêt à ce que se développe une critique des traductions, qui permettra entre autres de comprendre comment ont été vécus les rapports du traducteur à sa propre culture et à la culture étrangère, et par extension les rapports de la culture du Même à la culture de l'Autre. La critique passe par une analytique des textes traduits pour faire apparaître les modes de traduire et d'une certaine façon les modes d'être des cultures, et ainsi de mettre au jour la dynamique et la dialectique entre les deux. On fera inévitablement apparaître ainsi la perspective historique de la traduction. Car au delà des individualités au niveau des traducteurs, chaque culture, chaque époque (Renaissance, classicisme, romantisme, par exemple), marque le traduire de sa propre vision du monde. Le travail critique permettra ainsi de conscientiser le traducteur sur cet état de fait, ce qui ne manquera pas par contrecoup de poser le problème de l'inscription et de l'attitude du traducteur d'aujourd'hui dans l'interculturalité de son époque.

Il faut dire que nous vivons actuellement une crise de la critique, et tout particulièrement dans le domaine de la traduction. Non pas qu'il y ait absence de critiques. Mais plutôt parce que ces critiques n'obéissent à aucune règle, ne constituent pas un genre en soi, aisément identifiable, et caractérisé par une certaine autorité scientifique dans le domaine de la littérature. Antoine Berman tout en définissant ce qu'il entend par critique, constate cette insuffisance: «Mais si critique veut dire analyse rigoureuse d'une traduction, de ses traits fondamentaux, du projet qui lui a donné naissance, de l'horizon dans lequel elle a surgi, de la position du traducteur; si critique veut dire fondamentalement, dégagement de la vérité d'une traduction, alors il faut dire que la critique des traductions commence à peine à exister»(Berman 1995: 13-14)

La position (non polémique, soulignons-le) d'Antoine Berman sur la critique nous paraît féconde quant à notre problématique de l'interculturalité parce qu'elle resitue l'acte de traduire lui-même dans un champ plus vaste que ne le fait la tradition, mais un champ qui est vraiment le sien propre. D’abord cette idée de translation de l'œuvre inclut, comme nous l'avons vu, tout le travail de la critique et des «nombreuses formes de transformations de l'œuvre», textuelles ou non, traductives ou non. Mais il y a aussi tout le travail d'à côté qui accompagne l'œuvre. C'est ce qu'Antoine Berman appelle «l'étayage de la traduction »: «L'étayage de la traduction comprend tous les paratextes qui viennent la soutenir: introduction, préface, postface, notes, glossaires, etc. La traduction ne peut pas être "nue" sous peine de ne pas accomplir la translation littéraire. Aujourd'hui, les étayages traductifs proposés par l'âge classique, puis par l'âge philologique ( $\mathrm{XIX}^{\mathrm{e}}$ siècle), ne suffisent plus. Ils doivent être - et sont en train de l'être par certains traducteurs - repensés. La question de ces nouveaux 
étayages, et d'un nouveau consensus à ce propos, est d'une importance cruciale» (ibid.: 68$)^{9}$.

Il faut donc repenser aujourd'hui notre rapport aux textes traduits, et nous pourrons en tirer des conséquences pour la traduction d'aujourd'hui. D'autre part, sur le plan de la culture, ce concept d' «étayage de la traduction » nous paraît riche de possibilités pour aborder l'intraduisibilité. Nous avons en effet déjà abordé cette question à propos du non-dit culturel (Cordonnier 1995: 172-176). Le degré de traduisibilité est directement proportionnel au degré de fréquentation des cultures. Plus la fréquentation est faible plus le degré d'intraduisibilité paraîtra grand. Et comme dans la tradition occidentale, nous vivons sur le mythe de la transparence du traducteur, notion que nous avons en son temps critiquée (ibid.: 144-146 et passim), ce dernier n'ose pas sortir de l'ombre de l'auteur pour constituer l'à côté culturel de la traduction qui donnera à sa culture des clés pour entrer dans l'œuvre, et qui en même temps lui ouvrira des espaces de traduisibilité.

Il y a là une tradition de la traduction à retrouver, le traducteur construisant l'étayage de la traduction ${ }^{10}$, c'est-à-dire retrouvant d'une certaine manière son rôle de vulgarisateur qu'il a eu dans le passé, mais un rôle de vulgarisateur pensé dans la cohérence, dans le champ plus vaste de la translation des œuvres. Nous sommes donc devant un immense domaine de recherche où l'on pourra observer le rapport dialectique entre la vulgarisation des faits culturels et le travail consécutif sur la réduction de l'espace de l'intraduisibilité. Il faudra donc étudier tout le cheminement de l'œuvre à travers l'ensemble des textes traduits ou non, des paratextes la commentant, des adaptations, etc., car la traduction se situe dans ce creuset.

Antoine Berman ne peut que constater le manque d' "une théorie générale de la translation littéraire, du passage d'une ouvre d'une "langue-culture" à une autre» (ibid.: 56) ${ }^{11}$. Mais on voit bien toute la potentialité de cette voie qui est tracée pour avancer vers des propositions qui aideront les traducteurs à mieux affronter la traduction de la culture.

\section{Éthique}

Tous ces efforts de conceptualisation sont destinés à ce que le traducteur d'aujourd'hui puisse se situer clairement dans les rapports d'altérité. En effet, l'histoire de la traduction, les avancées réalisées dans le cadre des sciences humaines imposent des attitudes et des devoirs nouveaux. Notamment, dans les relations interculturelles, en ce qui concerne l'attitude face à l'Autre. Car cette attitude n'est pas sans avoir quelque influence dans les textes traduits eux-mêmes.

Faisons maintenant un petit retour en arrière. Si, dans Traduction et culture, nous avons fait la critique de l'ethnocentrisme en traduction, ce n'est pas tant pour ce que celui-ci était à l'époque, même si nous savons ce qu'il a pu induire comme souffrances humaines, que pour ce qu'il peut induire comme comportement chez les traducteurs aujourd'hui. Il y avait d'un côté un travail de conscientisation à mener sur les modes de traduire, et à se poser d'un autre côté la question de la place des traductions ethnocentriques aujourd'hui au sein de l'espace littéraire et culturel, dans le grand mouvement de translation des textes.

Il est évident qu'à l'aube du $\mathrm{XxI}^{\mathrm{e}}$ siècle, nous ne pouvons plus nous conduire face à l'Autre comme par le passé. Il y a d'abord des raisons morales, mais cet argument 
dépasse les limites de la présente étude, mais aussi et surtout le rôle constitutif sur le plan de la culture de la traduction a changé. On peut aussi inverser l'argument: notre culture dans son rapport au monde, aujourd'hui, a besoin d'être fécondée autrement par la traduction. C'est donc bien dans une perspective historique que nous nous plaçons. Selon nous, la traduction doit se situer maintenant au sein d'une éthique qui présidera au mouvement général de translation des œuvres, et plus spécifiquement au travail traductif en général.

Dans le cadre d'une éthique de la traduction, nous avons proposé le néologisme d'ouvertude (Cordonnier 1995: 153-154 et passim), pour qualifier l'attitude du traducteur dans la relation d'altérité. Ce néologisme fait partie du travail de conscientisation dont nous avons déjà parlé. Il est vrai que toutes les cultures sont plus ou moins ethnocentriques, à la différence près, et qui est quand même de taille, que certaines d'entre elles dominent alors que d'autres non. En France le classicisme a exacerbé le mouvement ethnocentrique, mais celui-ci a eu un rôle positif et fécondateur puisqu'il est un des éléments constitutifs essentiels de notre littérature et de notre culture. Cette force passée explique la vigueur des idées classiques sur la langue, sur la culture, et partant sur la traduction de nos jours encore ${ }^{12}$. Cette disposition héritée de l'histoire gêne la traduction dans son nécessaire redéploiement culturel. C'est donc pour sortir d'une pratique ethnocentrique trop généralisée, pour montrer clairement une autre voie, que nous avons forgé le concept d'ouvertude.

Il s'agit donc pour le traducteur qui choisit cette optique d'assumer une position traductive qui consiste à féconder la culture propre en faisant de la «montre » de la culture de l'Autre le fondement de son travail. C'est là son projet de traduction. Il s'agit, de traduction en traduction, d'apporter à sa culture des éléments culturels constitutifs nouveaux qui lui permettront peu à peu d'envisager les relations d'altérité plus sereinement, en meilleure connaissance de cause, et d'améliorer la communication et la compréhension interculturelles dans le monde de demain.

C'est pourquoi nous avons appelé à la traduction-dévoilement. Car il y a à retraduire les œuvres qui n'ont connu jusqu'à présent que des traductions annexées, et il y a aussi à donner au public contemporain des textes traduits qui laissent entrevoir la vérité de l'œuvre, étant entendu que cette vérité ne peut être que relative, en liaison aux outils conceptuels qui sont les nôtres actuellement. Mais ce retournement historique qui consiste à sortir de l'enfermement à l'intérieur du Même, enfermement qui était celui de l'ethnocentrisme, pour se situer en un point, non figé du reste, et se trouvant quelque part vers l'Autre, n'est pas un abandon de soi-même, de l'identité, comme cela a pu être reproché aux positions de Henri Meschonnic ou d'Antoine Berman. C'est ainsi que Jean-René Ladmiral va jusqu'à parler de «haine de soi»: «disons que nous y voyons un symptôme de la haine de soi qui nous semble être une maladie de la culture occidentale de notre temps» (Ladmiral 1997: 133) ${ }^{13}$.

Le trait nous semble quelque peu rapide. Au contraire, Antoine Berman est très mesuré sur cette question. L'auteur ne fait pas preuve d'esprit de système, il reconnaît la liberté du traducteur, il lui reconnaît même «tous les droits» $(1995: 93)^{14}$. Simplement, il situe le traduire dans le cadre d'une éthique. Ce qui signifie que le traducteur ne doit pas passer sous silence son intervention sur le texte original via la traduction. S’il manipule le texte (sans connotation péjorative), s'il déforme, s’il adapte, pour ne prendre que ces exemples, Antoine Berman considère qu'il doit l'annoncer et non le 
passer sous silence, car il faut que le lecteur soit averti du type de traduction auquel il a affaire.

En fait ce qui est en jeu ici, c'est la question de l'identité culturelle. Il est vrai qu'on assiste à une certaine mode identitaire. Denys Cuche (1996: 83) constate que celle-ci « est le prolongement du phénomène d'exaltation de la différence qui a surgi dans les années soixante-dix». On peut comprendre ce mouvement de pendule, mais en traduction mettre la différence sur le devant de la scène ou critiquer l'ethnocentrisme ne signifie en aucune façon attenter à l'identité. Ou alors il faut admettre que la critique n'est pas admise.

D’abord il faut dire que la notion d'identité n'est pas figée. Elle est diversifiée et fluctuante. Son élaboration est incessante. On ne peut pas la concevoir en dehors du rapport à l'Autre. Denys Cuche ajoute que «l'identité est un construit qui s'élabore dans une relation qui oppose un groupe aux autres groupes avec lesquels il est en contact» (ibid.: 86). C’est dire son importance cruciale en traduction. Il est clair que la traduction construit l'essence des cultures. Aujourd'hui, promouvoir un mouvement traductif qui sans a priori mettra en avant la différence culturelle, c'est apporter une pierre à la structuration contemporaine de l'identité. Il s'agit de lui donner les outils qui l'aideront à mieux se déployer dans le monde d'aujourd'hui et de demain. Il s'agit donc de rendre plus efficace la communication interculturelle, et partant, les relations entre cultures, selon le principe: plus je connaîtrai l'Autre dans ses textes, plus il me connaîtra dans mes textes, mieux nous nous comprendrons.

Quant à Henri Meschonnic, s'il critique un amour de la langue qui repose sur de vieux mythes, stérilisateurs quant à l'évolution heureuse de notre culture, comme la clarté, la pureté, le génie supposés de la langue française, c'est pour sortir celle-ci d'une passivité défensive et stérile. Ne plus tourner sur soi-même, mais intégrer positivement l'altérité. Pour cela Henri Meschonnic (1997: 210) propose « une transformation de l'identité par la diversité». Il ne s'agit donc pas de baisser les bras devant «les identités vacillantes», à supposer qu'elles le soient, dont nous parle Jean-René Ladmiral (1997: 133). C'est tout le contraire. Il s'agit de regarder le monde d'aujourd'hui bien en face et d'accompagner heureusement sa mutation.

S’il est souhaitable que se développe un large mouvement de traduction-dévoilement, c'est parce que nous avons constaté un manque dans notre culture, et parce que nous sommes conscient de son rôle novateur et fécondateur. Cela ne signifie pas que nous désirions que cet élan traductif soit exclusif de tout autre type de traduction. Au contraire. Nous avons indiqué plus haut l'intérêt que le traducteur aurait à retrouver ce rôle de vulgarisateur qu'il a perdu dans le passé. On peut continuer sur cette lancée et proposer de remettre à l'honneur, dans un premier temps dans l'institution scolaire, ces exercices qui ont fait partie du champ traductif dans notre histoire, nous voulons parler de l'imitation, de la paraphrase, du pastiche, de l'adaptation, par exemple. Mais quel que soit le type de traduction pratiqué et proposé, ce que nous voulons clairement, c'est que désormais il soit clairement affiché.

\section{Tâches}

Quant à la traduction-dévoilement, si elle se propose de montrer l'Autre, elle a aussi pour tâche, dans l'immense chantier des relations interculturelles, de constituer la culture du Même. En ce sens on peut dire qu'elle participe du phénomène ethno- 
centrique. Mais avec une grande différence: alors que dans le passé la constitution de soi se faisait largement par l'effacement, désormais la constitution de soi se fait aussi par la mise en évidence de l'Autre. Nous avons indiqué plus haut que l'ethnocentrisme est, à des degrés divers, de toutes les cultures. Mais, comme le formule Denys Cuche (1996: 116), dans la culture occidentale, il s'agit cette fois d'un ethnocentrisme dont on fera un «usage méthodologique». Denys Cuche (ibid.) cite ensuite Pierre Bourdieu: "Je suis convaincu qu'une certaine forme d'ethnocentrisme, si l'on désigne ainsi la référence à sa propre expérience, à sa propre pratique, peut être la condition d'une véritable compréhension: à condition bien sûr que cette référence soit consciente et contrôlée [...]. Il est plus difficile de reconnaître dans les autres, d'apparence si étrangers, un moi qu'on ne veut pas connaître. Cessant alors d'être des projections plus ou moins complaisantes, l'ethnologie et la sociologie conduisent à une découverte de soi dans et par l'objectivation de soi qu'exige la connaissance de l'autre ${ }^{15} »$. Un mouvement traductif se situant dans une ouvertude consciente et contrôlée n'est donc pas contradictoire avec la constitution de l'identité. Bien au contraire, il s'agit d'appréhender la dialectique du Même et de l'Autre, de l'identité et de la différence, dans un mouvement allant vers une conscience claire de leur essence et de leur interrelation, qui ne se fasse au détriment ni de l'une ni de l'autre des deux cultures en présence.

Nous terminerons en insistant sur le fait que ce rôle constitutif de la traduction sur le plan de la culture est infini. On comprendra mieux maintenant pourquoi elle a de formidables tâches devant elle. En cela, dans leur réalisation, elle participe de la finitude humaine et de la complétude du langage, qui sont, on le sait, infinies. Cette façon de voir repositionne d'une façon positive le problème de l'intraduisible qui n'est ainsi plus considéré comme une fatalité. Quant au travail de ré-écriture de la traduction, sur le plan linguistique, il n'est pas celui ou du sens, ou de la signifiance, mais celui de la totalité du signe, c'est-à-dire celui du sens et de la signifiance. $\mathrm{Ni}$ cibliste, ni sourcier. Dichotomie simpliste dans laquelle nous ne nous reconnaissons pas. Le travail de ré-écriture n'est pas une traduction de la langue, mais de ce que le discours fait de la langue. Dans un entre-deux, qui se love dans la relation entre les deux cultures, entre ce que dit le texte de l'Autre, et ce que je lui fais dire dans la mienne, dans un rapport de tension culturelle entre langue et discours, tension sans cesse changeante, insaisissable.

\section{NOTES}

1. Le présent article est la version revue et corrigée d'une conférence prononcée lors du: «Premier colloque international: Les aspects culturels de la traduction», organisé par l'Université technique de Yildiz, Istanbul (Turquie), 22-24 octobre 1997.

2. Nous nous donnons pour but ici d'approfondir et d'examiner sous un autre angle des notions que nous avons introduites ailleurs: cf. Jean-Louis Cordonnier, Traduction et culture, 1995.

3. Évidemment, «modes de traduire» est à comprendre ici au masculin.

4. Pour un panorama plus complet du concept de culture, nous renvoyons le lecteur à Cuche (1996).

5. Voir la première partie: Pour une archéologie de la traduction.

6. Signalons à ce propos la parution d'un nouvel ouvrage sur la période classique: La Traduction à l'âge classique, études réunies par Michel Ballard et Lieven D’hulst, Lille, Presses universitaires du Septentrion, 1996.

7. Cet article est rédigé par Jean Delisle, avec la collaboration de Pierre Cloutier.

8. Soul. par l'auteur.

9. Voir note $\mathrm{n}^{\circ} 70$. Soul. par l'auteur. 
10. À ne pas confondre avec «l'étayage de l'acte traductif» (Berman 1995: 68), terme qui désigne tout le travail de recherche documentaire du traducteur, toutes les lectures nécessaires à entreprendre pour réussir une traduction.

11. Soul. par l'auteur.

12. Sur les a priori classiques encore en vigueur: clarté, génie, pureté, par exemple, voir: Meschonnic (1997).

13. Soul. par l'auteur.

14. Soul. par l'auteur.

15. Cette citation est tirée de Pierre Bourdieu: «Entretien avec Alban Bensa: quand les Canaques prennent la parole», in Actes de la recherche en sciences sociales, $\mathrm{n}^{\circ}$ 56, mars 1985, p. 79.

\section{RÉFÉRENCES}

Berman, A. (1984): L'épreuve de l'étranger. Culture et traduction dans l'Allemagne romantique, Gallimard, coll. «Les Essais».

— (1995): Pour une critique des traductions: John Donne, Paris, Gallimard, coll. «Bibliothèque des idées".

CORdonnier, J.-L. (1995) : Traduction et culture, CREDIF/Hatier-Didier, Coll. LAL.

Cuche, D. (1996): La notion de culture dans les sciences sociales, Paris, Éditions La Découverte, Coll. Repères, $\mathrm{n}^{\circ} 205$.

Delisle, J. et J. Woodsworth (sous la dir. de) (1995): Les traducteurs dans l'histoire, Les Presses de l'Université d'Ottawa/Éditions UNESCO.

Foucault, M. (1966): Les mots et les choses, Paris, Gallimard, coll. «Bibliothèque des sciences humaines".

Ladmiral, J.-R. (1997): "Aspects interculturels de la traduction», in: Hommage à Hasan-Ali Yücel - La traduction: carrefour des cultures et des temps, sous la dir. du Prof. Dr Hasan Anamur, Istanbul, Université technique de Yildiz.

Mercier, D. (1995): L'épreuve de la représentation. L'enseignement des langues étrangères et la pratique de la traduction en France aux XVII ${ }^{e}$ et XVIII siècles, Besançon, Annales littéraires de l'Université de Besançon, $\mathrm{n}^{\circ}$ 589, Diffusion Les Belles Lettres.

Meschonnic, H. (1973): Pour la poétique II, Paris, Gallimard, 1973.

- (1997): De la langue française, Paris, Hachette.

Mounin, G. (1963): Les problèmes théoriques de la traduction, Paris, Gallimard, coll. «TEL», nº 5. 